\title{
Preliminary Design of An ALMA Band 10 Single-Chip Dual-Polarisation SIS Mixer
}

\author{
Boon-Kok Tan*† and Ghassan Yassin* \\ *Department of Physics (Astrophysics), University of Oxford, Keble Road, Oxford OX1 3RH, UK \\ ${ }^{\dagger}$ Contact: boonkok.tan@physics.ox.ac.uk, phone +44 1865273303
}

\begin{abstract}
We present the design of an integrated dualpolarisation superconductor-insulator-superconductor (SIS) mixer operating at ALMA Band 10 frequency range. All the RF components, including the orthomode transducer (OMT), the quadrature hybrid and the SIS mixer circuits, are designed as superconducting planar circuits to form a single planar mixer chip. The mixer circuits will be fabricated using standard thin film deposition technology on an ultra-thin silicon-on-insulator (SoI) substrate. In this paper, we discuss in detail the design of the various RF components that make up the complete dual-polarisation SIS mixer chip, and provide the predicted performance using rigorous electromagnetic modelling. The optimised designs of these components are then integrated in a superconducting quantum mixer circuit modelling (SuperMix) environment for assessing the performance of the heterodyne parameters of the mixer such as mixer gain and noise temperature.
\end{abstract}

\section{INTRODUCTION}

Most modern heterodyne receiver utilise two independent RF chains, one for each polarisation state, to obtain the polarisation information and to fully recover the strength of the astronomical signal [1]. By combining the two receiver chains using a compact dual-polarisation (2-pol) mixer, eliminating the need for RF beam-diplexing optics, we can potentially double or even quadruple the number of pixels within the same space in an existing receiver cabin. This translates to an increase in observation speed, fully utilise the available high-grade atmospheric condition which is crucial, especially for near- $\mathrm{THz}$ observations where good weather condition is scarce even at the best sites.

In this paper, we present the design of a 2-pol superconductor-insulator-superconductor (SIS) receiver with all the RF circuitry deposited on a single silicon-on-insulator (SoI) chip using planar superconducting circuit technology. The two singly-polarised SIS mixers are integrated via a planar orthomode transducer (OMT), connected to a submillimetre (sub-mm) feed horn [2]. The receiver is designed to operate from 787-950 GHz, in tandem with Atacama Large Millimetre/Sub-Millimetre Array (ALMA) Band 10 frequency range.

The 2-pol chip will be housed within a compact single split mixer block, thereby freeing up space in an existing receiver cabin such that an additional 2-pol receiver can be added. Alternatively, two different frequency bands can be combined in a single cartridge, allowing the telescope to access new science without substantial change of the telescope infrastructure. This could be applied to any existing millimetre and sub-

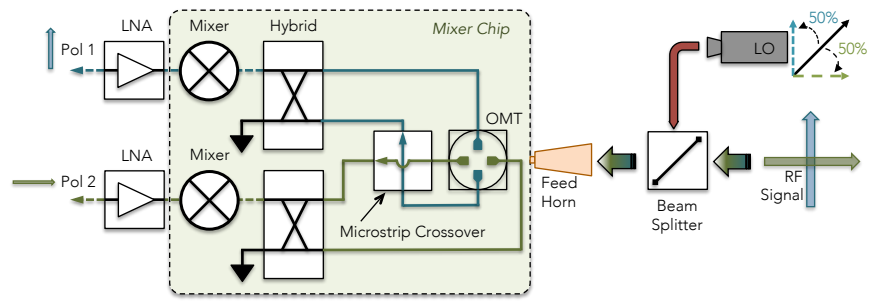

(a)

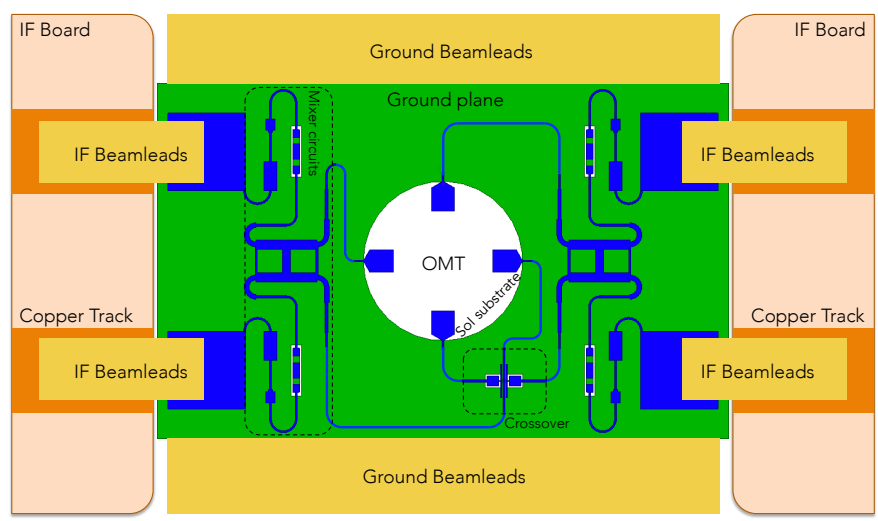

(b)

Fig. 1. (a) Schematic diagram illustrating the circuit components required for constructing a single-chip dual-polarisation SIS receiver. (b) The layout of the entire 2-pol mixer chip. The IF components e.g., the IF board and beam leads etc, are not drawn to scale to highlight the RF circuits for clarity purposes.

mm single-dish observatories e.g., Greenland Telescope (GLT) and Large Millimetre Telescope (LMT), or interferometric facilities which have access to high-grade weather condition, like Sub-Millimetre Array (SMA) or ALMA. Although, we focus on the design of an integrated 2-pol mixer operating at ALMA Band 10, the use of planar circuit technology means that the design can be scaled easily to other frequency bands as well.

\section{Single Chip Dual-Polarisation MiXer}

Fig. 1 shows a generic schematic assembly of the 2-pol receiver and the layout of the 2-pol mixer chip comprising the OMT, hybrids, mixer circuits and other ancillary components. The RF and the local oscillator (LO) signals are coupled to the mixer chip via a feed horn, where the incoming signal is split into two orthogonally polarised states through a quad-probe OMT in a circular waveguide [3], similar to the ones used 


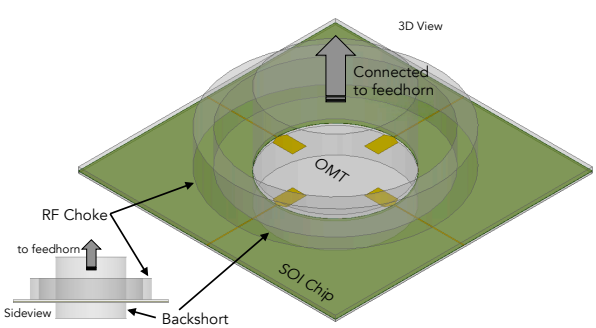

(a) Planar OMT

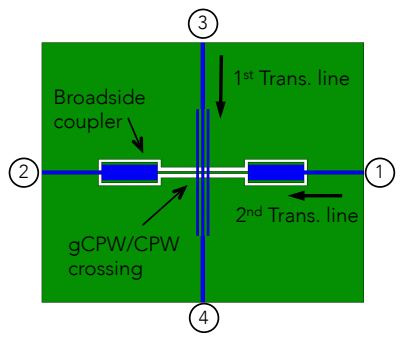

(b) Microstrip crossover

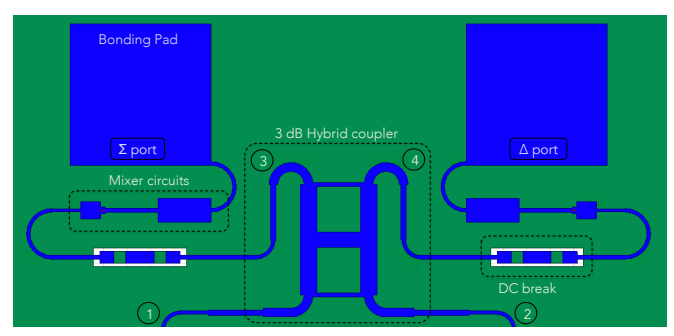

(c) Planar mixer circuits

Fig. 2. (a) A 4-probe OMT supported by a $3 \mu \mathrm{m}$ thick SoI substrate, including the circular RF choke. (b) Microstrip crossover with two orthogonally crossing CPWs. (c) The planar mixer circuit comprising a quadrature hybrid, DC breaks and SIS mixer tuning circuits.

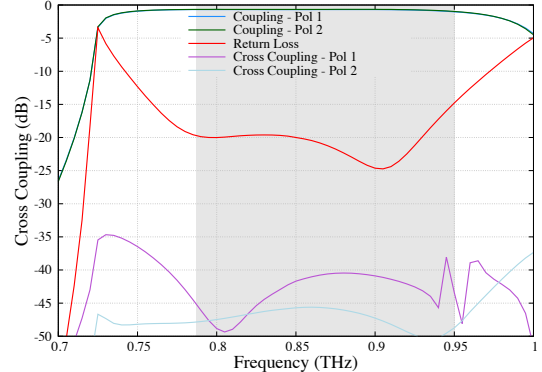

(a) OMT performance

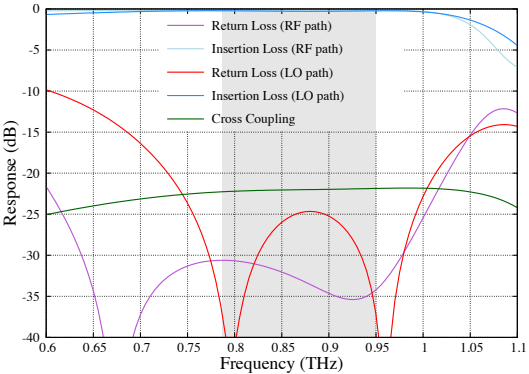

(b) Crossover performance

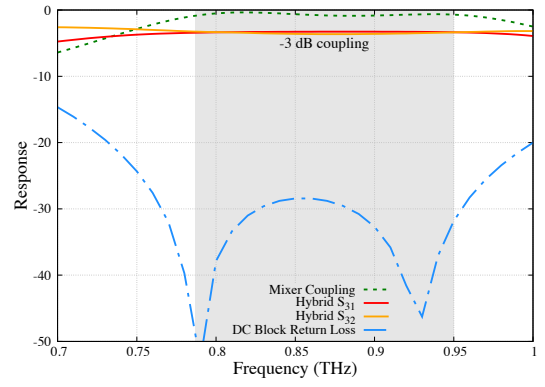

(c) Predicted responses

Fig. 3. The HFSS predicted performances of (a) the OMT, (b) the crossover and (c) the hybrid, DC block and the mixer's coupling coefficient.

in CMB bolometer experiments. The LO signal is injected to the feed horn via a free space beam splitter, with the E-plane aligned at an angle with the OMT axis, such that equal LO power is delivered to both polarisation states. For each state, each opposing pair of the probes pick up one half of the power of the input signal and then the signals from the two opposing probes are combined via the sum $(\Sigma)$ port of a hybrid, and fed to an SIS mixer for frequency down conversion. The difference $(\Delta)$ port of the hybrid contains only noise, and therefore can be terminated with an RF load. In our case, we utilise the SIS junctions biased above the gap as a resistive load [4].

In the following, we describe the individual key components required to form the mixer chip, along with their expected performances simulated using Ansys High Frequency Structure Simulator (HFSS), including the superconductivity effects. All RF circuit components, excluding the cross-over, are fabricated in microstrip lines with the $400 \mathrm{~nm}$ niobium nitride $(\mathrm{NbN})$ wiring and ground layers sandwiching a $475 \mathrm{~nm}$ thick silicon monoxide ( $\mathrm{SiO})$ dielectric layer. The entire planar circuitry is deposited on a $3 \mu \mathrm{m}$ thin SoI substrate, with gold beam leads for IF and ground connections.

Planar OMT: Broadband othormode transducer is a commonly used device to extract two orthogonally polarised modes from an incoming RF signal. Here, we use two sets of planar rectangular-probe antennas in a circular waveguide to perform this task. In Fig. 2(a), we show the design of a planar OMT operating in the frequency range of $787-950 \mathrm{GHz}$. Four thin-film microstrip-fed rectangular probes, two for each polarisation, are positioned transverse to the axis of a circular waveguide. A quarter wavelength backshort is used to optimise the coupling from the circular waveguide to the planar probes, and a radial waveguide choke is used to minimise the RF leakage through the groove that accommodates the planar circuit chip. As shown in Fig. 3(a), electromagnetic simulation performed using the HFSS suggests that the coupling from each of the polarised input to the corresponding pair of probes approaches $0 \mathrm{~dB}$ for both states. The return loss of the OMT is better than $-20 \mathrm{~dB}$, and the cross-coupling performance is also excellent, well-below $-40 \mathrm{~dB}$ across the entire operation bandwidth.

Microstrip Crossover: While the 2-pol planar OMT has been used in several B-mode CMB experiments, to our knowledge it has never been used in heterodyne receiver, probably because a microstrip crossing is required, as the two transmission lines emerging from the OMT must pass over each other to reach the mixers, as shown in Fig. 1(b). Clearly, crossing must be achieved without any significant coupling between the two lines, which is a challenge to achieve over a broad band without introducing the complexity of multiple layers photo-lithography. In contrast to the conventional approach which uses vias between microstrip line, we have introduced a simpler design that employs a pair of coplanar waveguides (CPW) aligned orthogonally on top of each other [5]. Due to the coplanar geometry, the field lines are transverse to the other, hence preventing signal leakage from one polarization to the other during the cross over. The transition from microstrip to the top CPW is straightforward as the structure can be deposited at the top-layer levelled with the microstrip, as illustrated in Fig. 2(b) (blue colour, 3-4 arm). The bottom CPW forming the lower transmission line is fabricated in the ground plane (green colour, 1-2 arm). The transition from microstrip to this CPW is achieved via a pair of broadside couplers. The structure is easy to fabricate, with clearly separated layers. The optimised performance is 


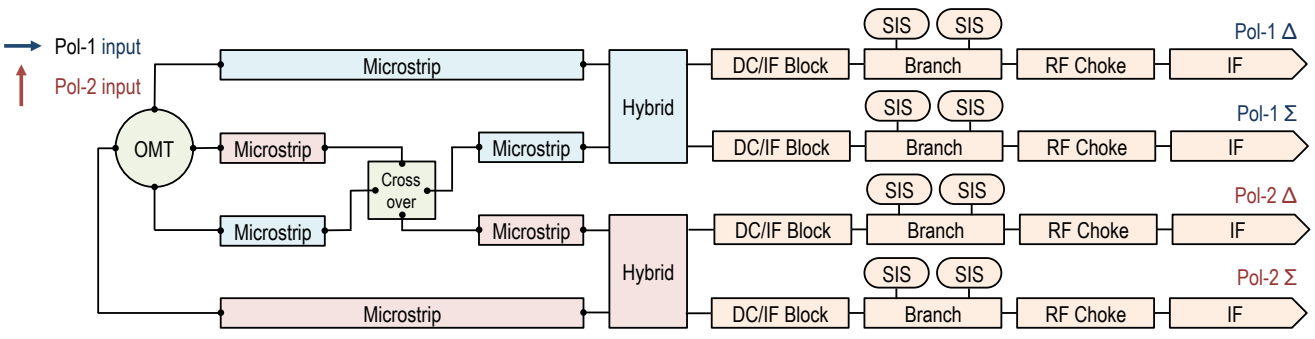

Fig. 4. Schematic diagram showing how the various RF components are combined within the Ansys Designer and SuperMix environments to simulate the performance of the entire 2-pol mixer chip.

broadband of more than $300 \mathrm{GHz}$ as shown in Fig. 3(b), with less than $-25 \mathrm{~dB}$ return loss and lower than $-22 \mathrm{~dB}$ crosscoupling over the required $787-950 \mathrm{GHz}$ band.

Quadrature Hybrid: To obtain the two orthogonally polarized modes, the signals from each pair of opposite probes need to be combined via a quadrature hybrid. Here we employ a design that cascades two microstrip hybrids for broadband response, as shown in Fig. 2(c), having the performance shown in Fig. 3(c) (solid red and orange line). The coupling from the output port 3 to the input port 1 (through) and port 2 (coupled) is closed to $-3 \mathrm{~dB}$, across the desired $787-950 \mathrm{GHz}$ band. Since a hybrid is a reciprocal device, this implies that the signals from the two planar probes of the same polarisation axis that feed to port 1 and port 2 correspondingly, will combined in-phase at port 3 . The power at the output port 4 is negligible since it contains only the difference $(\Delta)$ between the two input ports, and can be terminated with a load. Here, we use the SIS junction biased above the gap voltage as the resistive load. The performance of the quadrature hybrid has been examined before in our previous $650 \mathrm{GHz}$ balanced SIS mixer works [6], and the measured results showed good performance, agreeing well with theoretical predictions.

Mixer Circuits: Recent advances in device fabrication and mixer analysis has resulted in niobium $(\mathrm{Nb})$ based SIS mixer sensitivity approaching the quantum limit at frequencies below the superconducting gap frequency. It is well known however that the junction can still operate as a mixer well above the gap, although above the gap, the sensitivity of the mixer is degraded as a result of high conduction loss in $\mathrm{Nb}$ based transmission lines. One way to avoid this loss is to fabricate the mixer circuits using $\mathrm{NbN}$ line which has a much higher superconducting gap than $\mathrm{Nb}$. However, the use of $\mathrm{Nb}$ junction in conjunction with high-gap superconducting circuits have been shown to cause junction heating [7], due to Andreev reflection, where the thermalised quasi-particles are trapped in the $\mathrm{Nb}$ layer by the two higher-gap superconductors. We have therefore chosen to fabricate the top layer of all mixer circuits from normal metal (aluminium) to circumvent this problem, leaving the ground plane from $\mathrm{NbN}$ as in [8].

Our tuner circuit is simple, with just an end-loaded stub working in conjunction with a pair of tunnel junctions a quarter wavelength apart to tune out the parasitic junction capacitances [9], and a choke as an RF block. With this design, the signal coupling bandwidth of the mixer is broad from almost 0.75 $1 \mathrm{THz}$ with closed to $0 \mathrm{~dB}$ coupling, as shown in Fig. 3(c) (the dotted green line).
To avoid the interference and to allow separate biasing for all four mixer circuits (two for down-conversion, and two as resistive loads), we incorporate a DC break before each mixer, acting as bandpass filters. The DC break comprises of two cascaded broadside couplers, with the top and bottom layers separated by the dielectric, hence breaking the DC connection. The bandpass behaviour of the DC breaks is depicted as the blue dashed-dot-dashed line in Fig. 3(c), which shows very broadband performance with the return loss less than $-30 \mathrm{~dB}$ from 770-950 GHz.

\section{Heterodyne Performance of Full MiXer ChiP}

The full circuit of the entire 2-pol mixer chip is electrically too large for simulation in the HFSS environment. Therefore, in order to validate the feasibility of combining all the circuit components into an integrated circuit, we exported the $\mathrm{S}$ parameters of all components simulated using HFSS into a circuit model constructed using Ansys Designer software. Fig. 4 shows the equivalent circuit diagram of the full 2pol chip, with two separated polarisation branches shown in light blue (upper branch) and red (lower branch). The first polarisation signal (in blue) picked up by the first pair of the OMT probes is combined through the upper hybrid, via the crossover, before feeding the mixer for down conversion at the Pol-1 $\Sigma$ branch. The same applies to the second polarisation (red) signal path. The various microstrip line boxes represent the length of transmission line required to route around the OMT before connecting to the hybrid, with the length of the microstrip pairs connecting to the crossover altered slightly to compensate for the phase difference between the two crossing lines in the crossover. The mixer circuits comprising the DC block, SIS junctions and IF circuitries are the same for all four IF outputs (i.e., Pol- $1 \Delta$, Pol- $1 \Sigma$, Pol- $2 \Delta$, and Pol-2 $\Sigma$ ).

The simulated results are shown in Fig. 5. As can be seen, the coupling from both polarisation states to the mixer of both sum $(\Sigma)$ branches are closed to $-1 \mathrm{~dB}$ across the entire 787-950 GHz range. The return losses for both branches are also well below $-10 \mathrm{~dB}$ within the same range. Furthermore, the behaviour of both polarisation branches is largely similar, which is important for full recovery of the astronomical signal.

In order to study the heterodyne behaviour of the 2-pol mixer chip, we exported the same S-parameter sets to SuperMix [10], a superconducting mixer simulation code. The full SuperMix circuit model is similar to the one shown in Fig. 4, except that both the $\Delta$ branches after the hybrids were replaced for simplicity by resistive loads to terminate 


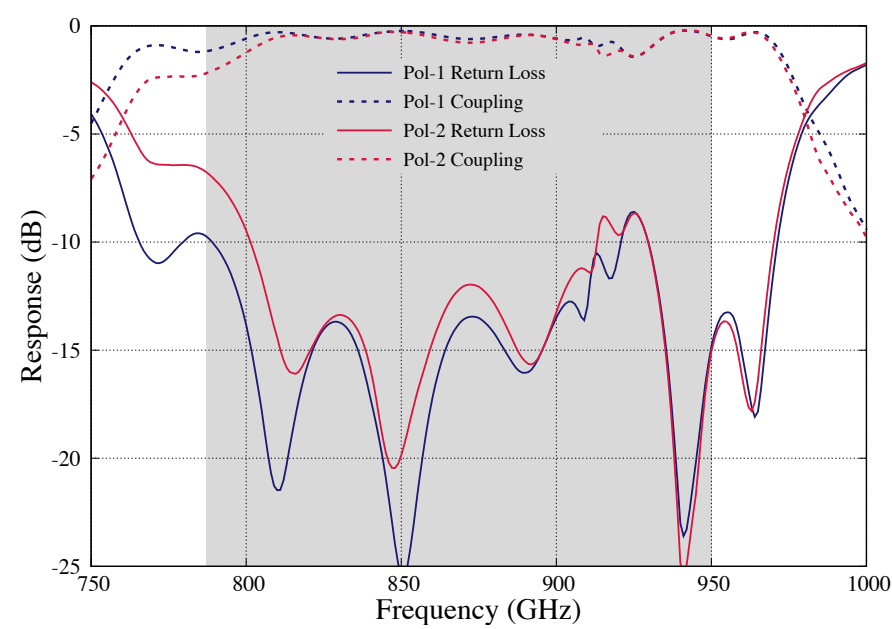

Fig. 5. The coupling from each polarisation to the corresponding SIS mixer, and the return losses, of the entire 2-pol chip simulated by Ansys Designer.

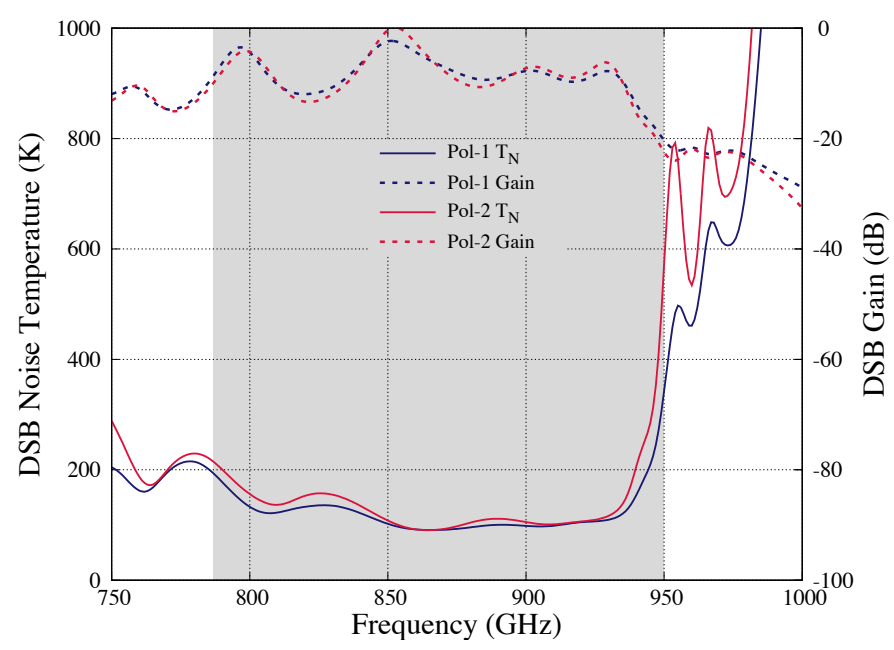

Fig. 6. The SuperMix predicted DSB gains and noise temperatures for both polarisation states of the entire dual-pol chip.

the isolated ports of the hybrids. Again, the simulated results show very promising performance, with the predicted double sideband (DSB) noise temperatures for both polarisation states closed to $100 \mathrm{~K}$ across the designated bandwidth, which is approximately $2.5 \times$ quantum limited sensitivity. The DSB gain from both polarisations is simulated to be around -5 to $-10 \mathrm{~dB}$, with a small variation within the band, as the mixer gain is often more sensitive to the losses in front of the mixer, as can be seen where the peak of the gain curves at $850 \mathrm{GHz}$ (approaching $0 \mathrm{~dB}$ ) corresponding to the lowest return loss in Fig. 5. In summary, the heterodyne performances for both polarisation states of the 2-pol mixer chips is similar across the band, giving good noise temperature. The gain variation across the band can certainly be improved by more careful design of the mixer circuits.

\section{CONCLUSION}

We have presented the design of a dual-polarisation SIS mixer chip where all the RF circuit components required to split the astronomical signal into two orthogonally polarised states are incorporated on-chip via the superconducting planar circuit technology. We first designed the individual RF components using rigorous 3-D electromagnetic simulators, and optimised the performance for operation at ALMA Band 10 range. Next, we integrated all these components within a circuit model to form the full mixer chip for validating the final performance of the full mixer chip. The electromagnetic response of the full mixer model shows good performance across the band. The full chip model was also constructed within the SuperMix environment to simulate the heterodyne behaviour of the mixer chip. The predicted mixer gains and noise temperatures for both polarisation states show promising results across the targeted bandwidth, approaching quantum limited sensitivity performance.

\section{REFERENCES}

[1] A. Baryshev, R. Hesper, F. Mena, T. Klapwijk, T. Van Kempen, M. Hogerheijde, B. Jackson, J. Adema, G. Gerlofsma, M. Bekema, et al., "The ALMA Band 9 receiver-Design, construction, characterization, and first light," Astronomy \& Astrophysics, vol. 577, p. A129, 2015.

[2] B.-K. Tan, J. Leech, G. Yassin, P. Kittara, M. Tacon, S. Wangsuya, and C. Groppi, "A high performance $700 \mathrm{GHz}$ feed horn," Journal of Infrared, Millimeter and Terahertz Waves, vol. 33, no. 1, pp. 1-5, 2012.

[3] P. Grimes, O. King, G. Yassin, and M. Jones, "Compact broadband planar orthomode transducer," Electronics Letters, vol. 43, no. 21, pp. 1146-1148, 2007.

[4] B.-K. Tan, Development of coherent detector technologies for submillimetre wave astronomy observations. Springer, 2015.

[5] A. Abbosh, S. Ibrahim, and M. Karim, "Ultra-wideband crossover using microstrip-to-coplanar waveguide transitions," IEEE Microwave and Wireless Components Letters, vol. 22, no. 10, pp. 500-502, 2012.

[6] B.-K. Tan, G. Yassin, P. Grimes, K. Jacobs, and S. Withington, "Preliminary measurement results of a $650 \mathrm{GHz}$ planar circuit balanced SIS mixer," IEEE Transactions on Terahertz Science and Technology, vol. 3, no. 1, pp. 32-38, 2013.

[7] A. Khudchenko, A. M. Baryshev, K. I. Rudakov, P. M. Dmitriev, R. Hesper, L. de Jong, and V. P. Koshelets, "High-gap Nb-AlN-NbN SIS junctions for frequency band 790-950 GHz," IEEE Transactions on Terahertz Science and Technology, vol. 6, no. 1, pp. 127-132, 2016.

[8] Y. Fujii, M. Kroug, K. Kaneko, A. Gonzalez, Y. Uzawa, T. Kojima, K. Kuroiwa, A. Miyachi, K. Makise, Z. Wang, et al., "Performance of the ALMA band 10 SIS receiver prototype model," IEEE Transactions on Applied Superconductivity, vol. 21, no. 3, pp. 606-611, 2011.

[9] V. Y. Belitsky, S. Jacobsson, L. Filippenko, and E. Kollberg, "Broadband twin-junction tuning circuit for submillimeter SIS mixers," Microwave and Optical Technology Letters, vol. 10, no. 2, pp. 74-78, 1995.

[10] J. Ward, F. Rice, G. Chattopadhyay, and J. Zmuidzinas, "SuperMix: A flexible software library for high-frequency circuit simulation, including SIS mixers and superconducting elements," in Proceedings, 10th 1999. 